bottom-and I think a very creditable depth too- they found that, while the surface of the water might be full of thesc calcareous organisms, the bottom was not. There they found that red clay so pathetically alluded to by my friend on the right [Commander Stewart, who replied for the Navy] as the material to which when glory called him he might be reduced. This red clay is a great puzzle-a great mystery-how it comes there, what it arises from, whether it is, as the director has suggested, the ash of foraminiferæ; whether it is decomposed pumice-stone vomited out by volcanoes, and scattered over the surface, or whether, lastly, it has something to do with that meteoric dust which is being continually rained upon us from the spaces of the universe-which of these causes may be at the bottom of the phenomenon it is very hard to say; it is one of those points on which we shall have information by-and-by. I will not detain you further with speaking of the matters of interest which have come out of this cruise of the Challenger; I will orly in conclusion remind you that work of this kind could by no possibility be done without the zealous aid of an intelligent executive. That is the first condition, but our thanks have already been rendered to the executive officers of the Challenger. In the second place, it could only have been done by the aid of such a scientific staff, composed of picked men as was sent out in the Challenger, such men as Buchanan, Murray, and Moseley, and Wild, and Suhm ; and I can hardly mention the name of the last gentleman without, in passing, lamenting that he alone of all the staff who left our shores,- - he who certainly was the last person we should have imagined we should not see again--that a man of his accomplishments and promise and geniality and lovability should be the only one not to be welcomed back by the friends who loved him, and by the country which would have been glad to adopt him. But, again, a work such as has been done by the Challenger could only have been effectively carrical out under the direction, not only of a man who intellectually knew what he was about, but whose moral qualities were such as to get the people with whom he was associated to work with him."

Prof. Huxley concluded by referring to the harmony which throughout prevailed among the staff of the Chatlenger.

"When men are shut up together in a limited society, whether it be a cathedral town or a ship, they begin to hate one another unless the bishop is a very wise person. In this case I do not doubt that the bishop was a very wise person, and I do not believe that the whole course of the Chalienger afforded occasion for any such triangular duels as one hears of in the novels of Captain Marryat."

Sir C. Wyville Thomson made a suitable reply to the toast, giving a brief account of the various operations of the Challenger, and referring to the great amount of work yet to be done ere all the results could be given to the world.

\section{PHOTOGRAPHIC PROCESSES 1}

$\mathrm{T} T$ is not my intention to enter into the history of any of the processes to which $I$ propose to call your attention to-night, as I somewhat dread to enter upon such controversial ground. Probably the demonstration of the production of photographic prints by various methods will be of greater interest than any history.

Astronomy was the religion of the world's infancy, and it can hardly be a matter of surprise that untutored yet inquiring minds, unaided by any distinct revelation, should have attributed to the glorious orb, the centre of our solar system, the possession of divine attributes, and as they gazed upon the wondrous effects of his magical painting, that they should have offered to him their adoration and worship, and carefully noted any phenomena

I Lecture by Capt. Abney, R.E., F.R.S., at the Loan Collection, South Kensington. due to him. Thus probably the first photographic action noticed would be at a very early period of human existence, when the exposure of the epidermis to his rays caused what is known to us as tan, whilst the parts of the body covered would remain of their pristine whiteness. A photographic action which would be remarked at a later date would be the fading of colours in the sunlight. Ribbons, silks, curtains, and similar fabrics of a coloured nature undergo a change in tint when exposed to it.

I have here a specimen of a pink trimming used by the fair sex, and the lady who presented me with it informed me that it was "a most abominable take in," as the colour "goes" after two days' wear. Her ideas on the subject and my own somewhat differed, for to me it presented a capital opportunity of using the material as a means for obtaining a photographic print in a moderate time. I have here two results of the exposure of this stuff to the sunlight: One was exposed beneath a negative of an anatomical subject, and we have the image represented as white upon a pink ground. The other subject is a map. An ordinary map was superposed over a square piece of the stuff, and placed in sunlight whilst in contact. We have in this case the lines of the map represented as pink on a white ground, from which the colour had faded.

The general opinion is, I believe, that the colour is given off somewhat similarly to the scent from a rose. Were this entirely the case, the light would not act as it does, but beneath the negative or map, the colour would bleach uniformly. The bleaching seems to be a really chemical change in the dye due to the impact of light. There are many other bodies besides dyes which change in light, and some of them are of the most unlikely nature. I had intended to show you to-night the change that takes place in glass by expusure to light for long periods. My friend, Mr. Dallmeyer, has in his possession specimens of brown and flint glass, which have markedly changed colour in those halves of the prisms purposely exposed to solar influences. In some cases there is a "yellowing " of the body, and in others a decided "purpling."

It is, however, only those bodies which change rapidly in the light that are utilised in photography. The most common amongst these are various compounds of silver, for they are peculiarly sensitive to the action of light. Nearly every silver compound is more or less changed by it, and when I say changed I mean altered in chemical composition. When we reflect what light is we can better understand its action. Light, as experiment, confirmed by mathematical investigation, tells us, is caused by a series of waves issuing from the luminous source, not, indeed, trembling in our tangible atmosphere, but in a subtler and infinitely less dense medium, which pervades all space, and which exists even in the interior of the densest solids and liquids. These waves of ether, as this medium is called, batter against and try to insinuate themselves amongst the molecules of any body exposed to their action, a good many millions of millions of them impinging every second against it. Surely it is not surprising to think, small though the lengths of these waves be, that this persistent battering should in some instances be able to drive away from each of the molecules some one of the atoms of which they are composed.

Take as a type that salt of silver which was, perhaps, the first known to change in the presence of light-silver chloride. For our purpose we may represent each of its molecules as made up of two atoms of silver locked up with two atoms of chlorine. Let us consider the action of the light on only one molecule. The waves strike against it energetically and persistently; the swing that the molecule can take up is not in accord with the swing of the ether. It is shaken and battered till it finally gives up one atom of chlorine; the vibration of the remaining two atoms of silver and one of chlorine are of a different period, and are not sufficiently in discord to cause a further elimination of an atom. The molecule which contains the two atoms of silver and one of chlorine is called a sub-chloride of silver or argentous chloride, and is of a grey violet colour. If, then, I place silver chloride (held in position by a piece of paper) beneath a body, part of which is opaque and part transparent, and expose it to sunlight, I shall find that where the opaque parts cover it, there the white chloride will remain unchanged, whilst on the portions beneath the transparent parts, the dark silver sub-chloride will have been formed. Of course were the paper, after removal of the body, to be further exposed to light, the image obtained would disappear, as a blackening over the whole surface would

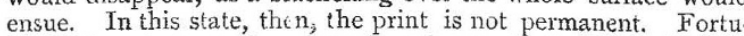
nately for photography, a ready solvent of silver chloride was 
found by Sir John Herschel in sodium-hyposulphite. On applying this salt to the inage, it was remored, and also one atom of silver and one of chlorine from the sub-chloride molecule, leaving the atom of metallic silver bshind. The chemical change that takes place on the silver chloride can be very distinctly shown by exposing it perfectly pure beneath water. The presence of the sub-chloride is shown by the colour, and that of the chlorine can be exhibited by the usual chemical tests.

In making an ordinary silver print on paper, we have, however, something more present than silver chloride; we have an organic salt known as the albuminate of silver, that is, a combination between albumen and silver. I have in this test-tube a little dilute albumen-the solid constituent of the white of an egg. Into it I drop a little silver nitrate : a flocculent precipitate is at once apparent. The silver from the nitxate has combined with the albumen, and on burning a piece of magnesium wire before it the outer surface shows a darkening; evidently, then, the albuminate of silver is decomposed by light. For silver printing purposes, paper is coated on one surface with a solution of albumen and sodium chloride, and the production of the silver chloride and albuminate is effected by floating that surface on a solution of silver nitrate. When dry, the paper which is now sensitive to light is ready for exposure beneath a negative. Here we have two prints produced on paper so prepared. If now I take one of them and dissolve away the insoluble salts in sodium hyposulphite, you see that the colour is of a disagreeable foxy-red tint. To show you how this want of a pleasing tone may be overcome, the other print is immersed in a weak solution of gold, and by a well-known chemical action the metaliic gold is deposited on the darkened portions of the picture. Now when gold is precipitated, it has not the well. known yellow colour, but is of a bluish purple; thus the depo. sited gold mixes its peculiar tint with that of the silver, and after immersion in the hyposulphite we obtain a print whose bearty cannot be surpassed.

I daresay that many of you may have been charmed with the production of magic photographs, as they were called. Some few years ago the sale of such was enormous, but now the curiosity of the public seems to be satiated. The magic, as you may be aware, consisted in being able to produce on a white piece of paper a photograph of some unknown object. These mysterious pieces of paper were generally supplied in packets, containing with them a piece of blotting-paper. The directions stated that the blotting-paper was to be damped, and whilst moist, to be applied to the surface of one of the accompanying pieces of blank paper, and then a photograph would shoot out. I will cndeavour to show you one method of their production. Fiere I have an ordinary photographic print which has not been treated with gold, but merely immersed in sodinm hyposulphite and then washed. I immerse it in a solution of mercurous chloride which I have in this dish, and immediately a bleaching action is set up. The action continues, and the paper is apparently blank. What has happened? Simply a white compound of silver and mercury has been formed, which is indistinguishable from the paper. If I wash the paper and dry it, it is in the state of the paper supplied in the packets. I have one here washed and dried, and I immerse it in the sodim hyposulphite. The image immediately reappears, a combination has taken place between the constituents of the hyposul. phide, the mercury, and the silver.

Need I say that the blotting-paper supplied is impregnated with the same sudium salt? In damping it the molecules of the iatter are so separated and mobile, that they are free to combine with the white image. By similar treatment the picture may be made to again disappear and once more reappear.

Besides silver there are varicus other metals which will give a photographic image. This paper, which has a slighily yellow tint, hes beei brushed over with ferric chloride, more commonly known as perchloride of iron, in which we have the maximum number of colours of chlorine combined with metallic iron. Allowing ordinary white light to act upon it, the waves cause a disturbance between the iron and the chlorine atoms, and one of the latter is shaken off, leaving ordinary ferrous chloride, or muriate of iron behind. A piece of paper, similariy prepared, has been exposed beneath a negative, and the reduction of the ferric chloride to the ferrous state can be demonstrated by floating it on a solution of potassium ferricyanide. The combination between the lowest type of the iron salt and this salt results in the formation of a deep blue precipitate known as 'Turnbull's blue. You see, after applying it, we have the lines of this map, of which this is the negative, of an intense blue.
Instead of demonstrating the change of the iron salt by this means, I may float it on a weak solution of silver nitrate. The ferrous salt of iron will reduce the silver, whilst the ferric salts are wholly inoperative to produce the same effect. Irere we have such a print.

The principal investigator of the action of light on iron com. pounds was Sir John Herschel, and he employed a variety of different combinations. Perhaps one of the most interesting exhibits in the Photographic Section is that old list of Fellow's of the Royal Society on which were pasted, by the hand of that distinguished philosopher, the actual solar spectrum prints mads during his researches on these and other metallic salts.

Uranium salts are also capable of being reduced to less complex forms by the action of light. I will not enter into a detailed description of the decomposition, but will simply exhibit the method of producing a print with the salt. The paper has been coated with uranic nitrate and exposed to light, beneath the same negative before shown to you. The image is made visible by a solution of potassium ferricyanide, as in the case of the iron salt.

In the cases of photographs are shown some interesting specimens of iron and uranium prints, made by Niè pce de St. Victor. I believe they were presented to Sir Charles Wheatstone by that ardent experimentalist. The subdued brewn tones of the latter were probably obtained by the admixture of a little iron with the uranium.

Within the last couple of years the salts of iron have been put to practical photographic printing purposes by $\mathrm{Mr}$. W. Willis, jun., of Birmingham, and a valuable process has resulted from his labours. The sensitive salt employed is an organic salt of iron known as ferric oxalate, and Mr. Willis made the discovery that amongst other metals platinum could be reduced to the metallic state from a double chloride of potassium and platinum, by ferrous oxalate in the presence of a potassic oxalate. A piece of paper is floated on a weak solution of silver nitrate and dried; and over the surface is brushed a mixture of the platinum salt and the ferric oxalate. After exposure to light (which produces the ferrous salts) beneath a negative, the paver is floated on a solution of neutral potassium oxalate, when the image at once appears formed of platinum black, a substance at once durable and incapable of being acted upon by atmospheiic influence. Such an exposed paper I have here, and floating it on oxalate solution, you see the image is imme. diately developed. The unreduced iron salt can be eliminated by soaking the print in the oxalate solution, and a rinse and hyposulphite removes all traces of silver nitrate. After a few changes of water, the print may be dried, and is permanent. I should explain that the paper is first coated with silver nitrate in order to cause the platintm to adhere firmly to the surface of the paper. When omitted, the fine black powder formed is apt to precipitate in the bath.

Before dwelling upon that metallic compound which in photo. graphy is next in importance to silver, I must call your atten. tion to the first vanadium print ever produced. Prof. Roscoe, who has already delighted an audience in this room with an admirable lecture on Dalton's apparatus and what he did with it, has made a classical investigation of the compounus of this metal, and amongst other interesting facts, has noticed that the vanadium salts are reduced by light in a somewhat similar manner to the uravium salts.

We now have to consider the printing processes which are due to the action of light on the dichromates of the alkalis in the presence of organic matter. For our purpose to-night we may take as a type potassium dichromate, a salt which readily parts with its oxygen to those compounds that have an avidity for it, more especially to certain carbon compounds under the influence of the ether waves.

To show that this salt is thus easily reducible by light in the presence of organic matter, I have here a piece of paper which has been brushed over with it, and exposed beneath a print. For a moment I float it on a weak solution of silver nitrate. The brilliant crimson colour of the part not exposed to light tells us that silver dichromate has been formed, but where the solar rays have acted, the colour remains unchanged. A slight modification of this process now exhibited to you is known as the chromatype, the offspring of Mr. Robert Hunt, so well known in the scientific world for his researches on light. Whilst experimenting with the chromatype process, Mr. W. Willis, the father of the gentleman I have already mentioned, discovered what is known as the aniline process. It is based on the fact that an acid in the presence of potassium 
dichrcmate strikes a blackish green or red colour when brought in contact with aniline. You will see the modus operandi when I say that paper is floated with potassium dichromate and a trace of phosphoric acid. Aniline is dissolved in spirits of wine, and the mixed vapours allowed to come in contact with the sensitive paper that has been exposed beneath a positive print, such as a map or plan. The impact of the light has so changed the potassium salt, that the aniline vapour causes but little coloration, whilst where the paper has been protected from it, the dark colour indicates that the dichromate is unchanged. The formation of this black colour is familiar to the manufacturers of aniline colours, being, I believe, similar in composition to the residue left after the formation of aniline purple by Mr. Perkins's method.

It shouli be noted that for copying engineers' tracings and drawings this process is extremely valuable, as there is no occasion to take a negative on glass before obtaining a print. All that is requisite is that the original should be fairly penetrable by light. A piece of paper prepared as indicated, a sheet of glass to place over the plan, and a box in which to place the exposed print to the aniline vapour are the only necessary plant for the reproduction of a design.

(To be continued.)

\section{NOTES}

Tre following are the officers of the forty-sixth annual meeting of the British Association which will commence at Glasgow on Wednesiay, September 6, 1876 :-President-designate--Prof Thomas Andrews, M.D., LL.D., F.R.S., Hon, F.R.S.E., in the place of Sir Robert Christison, Bart., M.D., D.C.L., F.R.S.E., who has resigned the Presidency in consequence of ill health. Vice-Presidents elect-Fris Grace the Duke of Argyll, K. T., F.R.S., \&c, the Lord Provost of Glasgow, Sir William Stirling Maxwell, Bart., M.A., M.P., Prof. Sir William Thomson, D.C.L., F.R.S., \&c., Prof. Allen Thomson, M.D., I.I.D., F.R.S., \&c., Prof. A. C. Ramsay, LL.D., F.R.S., \&c. Gencral Secretaries-Capt. Douglas Galton, C.B., D.C.L, F.R.S., \&c., Dr. Michael Foster, F.R.S. Assistant General Secrctary-George Griffith, M.A., F.C.S. General TreasurerProf. A. W. Williamson, Ph.D., F.R.S. Local SecretariesDr. W. G. Blackie, F.R.G.S., James Grahame, J. D. Marwick. Local Treasurers--Dr. Fergus, A. S. M'Clelland. The Sections are the following :--Section A : Mathematical and Physical Science. President-Prof. Sir W. Thomson, D.C.I., F.R.S. Section B: Chemical Science. President-W. H. Perkin, F.R.S. Section C: Geology. President-Prof. J. Young, M.D. Section D: Biology. President-A. Russell Wallace, F.L.S. Department of Anthropology, A. Russell Wallace, F.L.S. (President), will preside. Department of Zoology and Botany, Prof. A. Newton, F.R.S. (VicePresident), will preside. Department of Anatomy and Physiology, Dr. J. G. M'Kendrick (Vide-President), will preside. Section E: Geography. President-Capt. Evans, C.B., F.R.S., Hydrographer to the Admiralty. Section F: Economic Science and Statistics, President-Sir George Campbeil, K.C.S.I., M.P., D.C.L. Section G: Mechanical Science. President-C. W. Merrifield, F.R.S. The First Gencral Meeting will be beld on Wednesday, Sept. 6, at 8 p.m. precisely, when Sir John Hawkshaw, C.E., F.R.S., will resign the chair, and Prof. Andrews, F.R.S., President Designate, will assume the Presidency, and deliver an Address. On Thursday evening, Sept. 7 , at 8 p.m., there will be a soirce; on Friday evening, Sept. 8, at 8.30 p.m., a Discourse; on Monday evening, Sept. II, at 8.30 p.m., a Discourse by Prof. Sir C. Wyville Thomson, F.R.S. ; on Tuesday evening, Sept. I2, at 8 p.m., a soircé; on Wednesday, Sept. I3, the Concluding General Meeting will be held at 2.30 p.m. The Local Committec, as our readers will have seen from a previous report, have made unusual exertions to render the Glasgow meeting a success. A variety of interesting collections will be exhibited, and the excursions which bave been already arranged for will doubtless form one of the most attractive, and not the least instructive, feature of the meeting.

IT is with sincere regret that we notice the announcement in L'Explorateur of the death of the eminent and well-known geographer, Dr. August Heinrich Petermann, at the early age of fiftyfour years. He was born April is, 1822, at Bleicherode, in Prussian Saxony. In 1839 he became a pupil of the special Academy founded at Potsdam by the geographer Berghaus, whose secretary and librarian he was for six years, as well as collaborateur, for he took an active part in the preparation of the great Physical Atlas of his master; the English edition, which appeared at Edinburgh in 1847 , even bore his name. In 1845 he left Germany for Edinburgh, after two years' stay in which city he went to London, where he became a Fellow of the Royal Geographical Society. He wrote many valuable articles on the Progress of Geography, in the Atheneum and the "Encyclopædia Britannica," published the "Attas of Physical Geography" in conjunction with the Rev. Thomas Milner, and a Tableau of Central Africa according to the most recent explorations. It was greatly due to his influence that the English Government entrusted to the German travellers Barth, Overweg, and Vogel, missions fruitful in results both to science and commerce. Petermann also, as our readers know, paid great attention to questions connected with the Arctic regions, clough his opinions on certain points connected with Arctic geography are not likely to be confirmed. Still he did excellent service in this department by advocating the equipment of expeditions private and governmental, and by recording speedily and accurately the results from time to time obtained. In 1854 , Fetermann accepted the chair of geography in the University of Gotha, and in 1855 received from the University of Göttingen the degres of Ph.D. It was at this time that he undertook the direction of the great geographical establishment of Justus Perthes, of Gotha, and commenced to edit the well-known Mittheilungens, the monthly geographical review, whose scientific value has been long recognised. Petermann had a comprehensive idea of what is included under geographical science, and it will be difficult to supply his place either as editor of the Mittheilungen, or in the department of scientific geography.

Mr. Cross on Monday received a very numerous deputation from the British Medical Association, who laid before him their views with regard to the Vivisection Bill now before Parliament. These opinions were conveyed by Mr. Ernest Hart, Mr. John Simon, Dr. Wilks, senior physician of Guy's Hospital, and Sir W. Jenner, who raised his voice against a measure which would place men of science under police supervision, and would lay a ban upon them for inflicting cruelties on the lower animals when ten thousand times greater crueliies were inflicted by those who were going to pass this Bill. Such conduct would make those who passed it objects of scorn to all the scientific men in Europe. The Home Secretary, in reply, pointed out that the Bill was framed practically in accordance with the views of the Royal Commission, and that whether the Bill passed now depended entirely upon the line of conduct pursued by the medical pro. fession.

WE are compelled by a pressure on our space to postpone the continuation of Dr. Richardson's artlcles till next week.

THE Kew museums have recently acquired some interesting additions to their already unique and valuable collections by the presentation, by his Royal Highress the Prince of Wales, of the botanical specimens collected during his recent visit to India. These specimens consist of a number of seeds and fruits of economic or medicinal value, as well as of condiments, drugs, gums, \&c., from Southern India, and a series of named woods from Kanara. Though most of the seeds, fruits, and gums are already contained 